\title{
On the Statistics of the Sum of Correlated Generalized-K RVs
}

\author{
Saad Al-Ahmadi \\ Department of Systems and Computer Engineering \\ Carleton University \\ Ottawa, Canada \\ Email: saahmadi@sce.carleton.ca
}

\author{
Halim Yanikomeroglu \\ Department of Systems and Computer Engineering \\ Carleton University \\ Ottawa, Canada \\ Email: halim@sce.carleton.ca
}

\begin{abstract}
Appropriate channel modeling plays an important role in the design and analysis of various transmission and reception schemes over composite fading channels. The generalized$K$ (Gamma-Gamma) composite fading model has been used recently to model composite fading in wireless channels as an alternative to the less tractable lognormal-based models. In this paper, the expression of the amount of fading for the sum of correlated generalized- $K$ random variables is derived and then the moment matching method is used to approximate, in the lower tail region, the probability density function of the sum of identically distributed generalized- $K$ random variables with positively and equally correlated shadowing components by the familiar Gamma distribution. Furthermore, the obtained expressions of the amount of fading give insights into the effect of shadowing correlations on the performance of maximal ratio combining receivers in coordinated multi-point transmission and reception schemes in future wireless systems.

Index Terms-Composite fading, generalized-K model, multipath fading, shadowing, shadowing correlations, amount of fading, diversity gain, distributed antenna systems.
\end{abstract}

\section{INTRODUCTION}

The emergence of distributed antenna systems (DASs) and multi-hop relay networks has motivated revisiting the modeling of composite fading channels, since the design and analysis of such systems require developing appropriate and yet tractable models. In the past, the joint large-scale and small-scale fading performance analysis has been conducted using the lognormal-based composite fading models. Recently a more tractable model, the generalized- $K$ model, has received interest [1-4] since it has led to a closed-form expression for the instantaneous power probability density function (PDF) in shadowed Nakagami fading channels.

The sum distribution statistics are essential for the performance analysis of diversity schemes; however, further derivations on the distribution of the sum of generalized- $K$ random variables (RVs) have shown to be involved even for the independent and identically distributed (i.i.d.) case [5], and up the knowledge of the authors, no results have been reported so far for the correlated case (one exception is the simple special case mentioned in [6] where the shadowing components are assumed to be fully correlated, similar to

Saad Al-Ahmadi's work was supported by King Fahd University of Petroleum and Minerals, Saudi Arabia. previous formulation for the lognormal-based model [7]). So, it is of interest to develop closed-form approximations for the distribution of the sum of correlated generalized- $K$ RVs. The relevance of this problem stems from the fact that the shadowing components are expected to be correlated across the geographically distributed ports in DASs, base stations (BSs) in a multi-cell processing cellular system, or cooperating nodes in a multihop network. The shadowing components have a larger de-correlation distance in comparison to the multipath components since shadowing is caused by terrain configuration and/or large obstacles between the transmitter and receiver [8].

In this paper, first, the expression of the correlation coefficient between two generalized- $K$ RVs is derived in terms of the correlations coefficients between their multipath components and their shadowing components; then subsequent expressions of the amount of fading (AF) for the sum of correlated generalized- $K$ RVs are developed. Secondly, it is shown that the distribution of the sum of a moderate number of identically distributed generalized- $K$ RVs with positively and equally correlated shadowing components can be closely approximated region-wise by a Gamma PDF using an adjustable form of the expressions obtained by moment matching method. The obtained AF expressions can be used, utilizing the relations developed in [9-10] between AF and other performance metrics, to study the effect of shadowing correlations on those metrics. Using the approximate Gamma PDF, the performance analysis of maximal ratio combining (MRC) schemes and the computation of the probability of outage, the outage capacity, and the ergodic capacity of DASs and multi-hop relay networks, can be carried out. Moreover, the diversity gain can be predicted as the AF of the approximating Gamma model [11].

\section{The Generalized- $K$ Model}

The Nakagami- $m$ distribution is widely used to model the multipath fading statistics due to its applicability in different propagation environments. The PDF of the instantaneous power in a Nakagami multipath fading channel, conditioned on the local mean power $\Omega$, follows a Gamma PDF as

$p_{\gamma / \Omega}(x)=\frac{\left(\frac{m_{m}}{\Omega}\right)^{m_{m}}}{\Gamma\left(m_{m}\right)} x^{m_{m}-1} \exp \left(-\frac{m_{m} x}{\Omega}\right), x \geq 0, m_{m} \geq 0.5$. 
Although the variations in the local mean power are usually modeled by the lognormal distribution, the Gamma distribution was suggested in $[1-2,12]$ as a more tractable model that closely approximates the lognormal model for the relevant shadowing severity in wireless channels [3]

$$
p_{\Omega}(y)=\frac{\left(\frac{m_{s}}{\Omega_{0}}\right)^{m_{s}}}{\Gamma\left(m_{s}\right)} y^{m_{s}-1} \exp \left(-\frac{m_{s} y}{\Omega_{0}}\right), y \geq 0, m_{s}>0 .
$$

Based on the assumption that the fading and shadowing processes are independent due to the fact that the two phenomena are due to independent propagation mechanisms, the PDF of the instantaneous power $\gamma$ in a composite fading channel can be expressed, using the generalized- $K$ (Gamma-Gamma) model, as [1]

$$
p_{\gamma}(x)=\frac{2(b)^{m_{m}+m_{s}}}{\Gamma\left(m_{m}\right) \Gamma\left(m_{s}\right)} x^{\left(m_{m}+m_{s}\right) / 2-1} K_{m_{s}-m_{m}}(2 b \sqrt{x}),
$$

where $\Gamma[\cdot]$ is the Gamma function, $m_{m}$ and $m_{s}$ are the Nakagami multipath fading and shadowing parameters, respectively. In (3), $K_{m_{s}-m_{m}}(\cdot)$ is the modified Bessel function of the second kind and order $m_{s}-m_{m}, b=\sqrt{\frac{m_{m} m_{s}}{\Omega_{0}}}$ and $\Omega_{0}$ is the average power. The parameters $m_{m}$ and $m_{s}$ quantify the severity of multipath fading and shadowing in the sense that the severity of multipath fading and shadowing are inversely proportional to the values of $m_{m}$ and $m_{s}$, respectively ${ }^{1}$.

Remark 1: In fact, the distribution of the product of $M$ independent Gamma RVs, where the generalized- $K$ PDF corresponds to the case $M=2$, was derived in [13] in terms of the Meijer's function. Hence, the corresponding cumulative distribution function (CDF) and the characteristic function can also be expressed in terms of the Meijer's function.

Definition [14]: The amount of fading (AF) of the instantaneous power $\gamma$ is defined as the ratio of the variance to the square of the mean

$$
\mathbf{A F}=\frac{\operatorname{var}(\gamma)}{[E(\gamma)]^{2}},
$$

where $E(\cdot)$ and $\operatorname{var}(\cdot)$ denote the expectation and the variance of $\gamma$, respectively. The mean and the variance of a generalized$K \mathrm{RV}$ can be expressed as [4]

$$
\begin{gathered}
E(x)=\Omega_{0}, \\
\operatorname{var}(x)=\frac{\left(m_{m}+m_{s}+1\right) \Omega_{0}^{2}}{m_{m} m_{s}} .
\end{gathered}
$$

Now, using (5) and (6), the AF of $\gamma$ for the generalized- $K$ composite fading model can be expressed as

$$
\mathrm{AF}_{\gamma}=\frac{1}{m_{m}}+\frac{1}{m_{s}}+\frac{1}{m_{m} m_{s}} .
$$

\footnotetext{
${ }^{1}$ The parameter $m_{s}$ can be related to the standard deviation of the lognormal shadowing model as in $[3,12]$.
}

\section{THE AF FOR THE SUM OF CORRELATED GENERALIZED- $K$ RVS}

The sum of $N$ correlated generalized- $K$ RVs can be written, using the fact that each generalized- $K \mathrm{RV}$ is the product of two Gamma RVs, as

$$
\xi_{1}=z_{1} w_{1}+z_{2} w_{2}+\ldots+z_{N} w_{N},
$$

where $z_{i}$ and $w_{i}$ denote the multipath fading and shadowing $\mathrm{RVs}$, respectively. In general, the multipath components and the shadowing components are independent, but correlations among the $z_{i}^{\prime}$ s and among the $w_{i}^{\text {'s }}$ may exist in physical scenarios as discussed before.

In order to derive the expression of the AF for the sum of correlated generalized- $K$ RVs, first we need to develop the expression of the correlation coefficient between two generalized- $K$ RVs in terms of the correlation coefficients between their multipath and shadowing components. The relevance of revealing the individual roles of the multipath correlations and the shadowing correlations is motivated by the fact that these correlations take place at two different spatial scales as discussed before.

Lemma 1: The correlation coefficient between two identically distributed generalized- $K \mathrm{RVs}, \rho_{i, j}$, can be expressed in terms of the correlation coefficient between their multipath components $\left(\rho_{z_{i}, z_{j}}\right)$ and the correlation coefficient between their shadowing components $\left(\rho_{w_{i}, w_{j}}\right)$ as

$$
\rho_{i, j}=\frac{\rho_{z_{i}, z_{j}} m_{s}+\rho_{w_{i}, w_{j}} m_{m}+\rho_{z_{i}, z_{j}} \rho_{w_{i}, w_{j}}}{m_{m}+m_{s}+1} .
$$

Proof: Let us consider any two RVs of the summand in (8) as $\xi_{i}=z_{i} w_{i}$, and $\xi_{j}=z_{j} w_{j} ; \forall i, j=1, . ., N$; then, we may write the correlation coefficient between the $i$ th and the $j$ th generalized- $K$ RVs $\rho_{i, j}$ as

$$
\rho_{i, j}=\frac{E\left[z_{i} w_{i} z_{j} w_{j}\right]-E\left[z_{i} w_{i}\right] E\left[z_{j} w_{j}\right]}{\sigma_{z_{i} w_{i}} \sigma_{z_{j} w_{j}}} .
$$

Since the multipath components are independent from the shadowing components, the term $E\left[z_{i} w_{i} z_{j} w_{j}\right]$ can be expressed as

$$
E\left[z_{i} w_{i} z_{j} w_{j}\right]=E\left[z_{i} z_{j}\right] E\left[w_{i} w_{j}\right] .
$$

Substituting (11) in (10), and using $E\left[z_{i} z_{j}\right]=\rho_{z_{i}, z_{j}} \sigma_{z_{i}} \sigma_{z_{j}}+$ $E\left[z_{i}\right] E\left[z_{j}\right]$ and $E\left[w_{i} w_{j}\right]=\rho_{w_{i}, w_{j}} \sigma_{w_{i}} \sigma_{w_{j}}+E\left[w_{i}\right] E\left[w_{j}\right]$, result in

$$
\begin{aligned}
& \rho_{i, j}= \\
& \frac{\left[\rho_{z_{i}, z_{j}} \sigma_{z_{i}} \sigma_{z_{j}}+E\left[z_{i}\right] E\left[z_{j}\right]\right]\left[\rho_{w_{i}, w_{j}} \sigma_{w_{i}} \sigma_{w_{j}}+E\left[w_{i}\right] E\left[w_{j}\right]\right]-E\left[z_{i} w_{i}\right] E\left[z_{j} w_{j}\right]}{\sigma_{z_{i}} w_{i} \sigma_{z_{j}} w_{j}} .
\end{aligned}
$$

Now, using the interpretation of the generalized- $K$ composite fading RV as a multiplication of two Gamma RVs where the mean of the one corresponding to multipath fading is unity and the mean of the one corresponding to the shadowing is $\Omega_{0}$, we may write; $E\left[z_{i}\right]=1, \sigma_{z_{i}}=\sqrt{\frac{1}{m_{m, i}}}, E\left[w_{i}\right]=\Omega_{0, i}, \sigma_{w_{i}}=$ $\sqrt{\frac{\Omega_{0, i}^{2}}{m_{s, i}}}, E\left[z_{i} w_{i}\right]=\Omega_{0, i}$, and $\sigma_{z_{i} w_{i}}=\sqrt{\frac{\left(m_{m, i}+m_{s, i}+1\right) \Omega_{0, i}^{2}}{m_{m, i} m_{s, i}}}$. With the substitutions, (12) becomes 
$\rho_{i, j}=\frac{\rho_{z_{i}, z_{j}} \sqrt{m_{s, i} m_{s, j}}+\rho_{w_{i}, w_{j}} \sqrt{m_{m, i} m_{m, j}}+\rho_{z_{i}, z_{j}} \rho_{w_{i}, w_{j}}}{\sqrt{\left(m_{m, i}+m_{s, i}+1\right)} \sqrt{\left(m_{m, j}+m_{s, j}+1\right)}}$

The expression in (13) simplifies to the one in (9) for the identically distributed case.

Lemma 2: The AF for the sum of identically distributed and equally correlated (i.d.e.c.) $N$ generalized- $K$ RVs can be expressed as

$\mathrm{AF}_{\xi_{1}}^{\text {i.d.e.c }}=\frac{1-\rho_{m}}{N m_{m}}+\frac{1-\rho_{s}}{N m_{s}}+\frac{1-\rho_{m} \rho_{s}}{N m_{m} m_{s}}+\frac{\rho_{m}}{m_{m}}+\frac{\rho_{s}}{m_{s}}+\frac{\rho_{s} \rho_{m}}{m_{s} m_{m}}$ where $\rho_{m}=\rho_{z_{i}, z_{j}}$ and $\rho_{s}=\rho_{w_{i}, w_{j}} \forall i, j=1, \ldots, N$.

Proof: The AF for the sum of $N$ correlated generalized- $K$ RVs can be expressed as

$$
\mathrm{AF}_{\xi_{1}}=\frac{\sum_{i=1}^{N} \mathrm{AF}_{i} \Omega_{0, i}^{2}+\sum_{i=1}^{N} \sum_{j=1, i \neq j}^{N} \rho_{i, j} \sqrt{\mathrm{AF}_{i}} \sqrt{\mathrm{AF}_{j}} \Omega_{0, i} \Omega_{0, j}}{\left(\sum_{i=1}^{N} \Omega_{0, i}\right)^{2}} .
$$

Assuming i.d.e.c. $\left(\rho_{i, j}=\rho\right)$ generalized- $K$ RVs, the expression in (15) simplifies to

$$
\mathrm{AF}_{\xi_{1}}=\frac{(1+(N-1) \rho) \mathrm{AF}_{0}}{N}=\frac{(1-\rho)}{N} \mathrm{AF}_{0}+\rho \mathrm{AF}_{0},
$$

where $\mathrm{AF}_{0}$ denotes the $A F$ of each of the individual summands as given in (7). (14).

Now, using Lemma 1, we may substitute (9) in (16) to get

The results in Lemmas 1 and 2 have several interesting implications. First, the expression in (9) quantifies the individual role of the multipath correlation and the shadowing correlation in the overall correlation between the compositely faded signals, and consequently, in the expression of the AF for the sum of $N$ i.d.e.c. generalized- $K$ RVs. Secondly, the result in (14) indicates the existence of a non-vanishing part of $\mathrm{AF}_{\xi_{1}}$ with respect to increasing $N$, in correlated composite fading channels (in general, any correlated fading channel). The existence of this term explains why a correlated composite fading channel can not approach a non-fading channel $(\mathrm{AF}=0)$ as $N \rightarrow \infty$, since $\mathrm{AF}_{\xi_{1}}$ is always lower bounded as $\mathrm{AF}_{\xi_{1}} \geq \rho \mathrm{AF}_{0}$.

The case where $\left(\rho_{s}>0\right.$ and $\left.\rho_{m} \approx 0\right)$ represents a real scenario in both wireless channels and radar scattering when the fast fading components (multipath or speckle) are uncorrelated while the slow fading (shadowing) components are correlated, the corresponding expression of the $\mathrm{AF}$ is

$$
\mathrm{AF}_{\xi_{1}}^{i . d . e . c .}=\frac{1}{N m_{m}}+\frac{1-\rho_{s}}{N m_{s}}+\frac{1}{N m_{m} m_{s}}+\frac{\rho_{s}}{m_{s}} .
$$

The expression in (17) indicates (i) the AF due to the multipath component will vanish as $N \rightarrow \infty$ and the non-vanishing part (as $N \rightarrow \infty$ ), as expected, is due to correlated shadowing. However, that non-vanishing part, with respect to increasing
$N$, vanishes as $m_{s} \rightarrow \infty$ due to the fact that the Gamma PDF, corresponding to the shadowing component, approaches a Dirac-delta PDF as $m_{s} \rightarrow \infty$ so that the resulting composite fading PDF will be mainly the Gamma PDF associated with the power of the multipath fading component, and (ii) the existence of negative shadowing correlations results in reduced AF. This is further investigated in the next remark.

Remark 2: The existence of negative shadowing correlations was reported in some propagation scenarios [15]. So, it is of relevance to analytically investigate the effect of such correlations. The expression in (17) shows that negative shadowing correlation tends to decrease the non-vanishing term and increase the part of the AF that vanishes as $N \rightarrow \infty$. The gain of the existence of negative correlations was reported in [15].

Considering the case of $N=2$, the AF with negative correlations becomes

$$
\mathrm{AF}_{\xi_{1}}^{\text {i.d.e.c. }}=\frac{1}{2 m_{m}}+\frac{1-\rho_{s}}{2 m_{s}}+\frac{1}{2 m_{m} m_{s}}+\frac{\rho_{s}}{m_{s}}
$$

which shows that the effect of shadowing disappears for $\rho_{s}=$ -1 as expected. For $N=3$, the positive semi-definiteness (PSD) condition for a $3 \times 3$ correlation matrix can be expressed, using the fact that a necessary and sufficient condition for a symmetric $P \times P$ matrix, $A$, to be positive semidefinite is that all the possible principal minors of $\boldsymbol{A}$ are non-negative [16], as $1+2\left(\rho_{1,2} \rho_{1,3} \rho_{2,3}\right)-\left|\rho_{1,2}\right|^{2}-\left|\rho_{1,3}\right|^{2}-\left|\rho_{2,3}\right|^{2} \geq 0$ using this inequality, the minimum possible value of $\rho_{s}$ is -0.5 which will also result in canceling the effect of shadowing. For $N=4$, it can be found, numerically, that the corresponding value is $-1 / 3$. In general, it can be proved (using [17, problem 6.2.14(b)]) that the maximum allowable value, due to the PSD constraint, is $\rho_{s, \max }=-1 /(N-1), N>1$; hence $\rho_{s, \max }$ approaches zero as $N \rightarrow \infty$. Physically, this takes place since the AF can not be less than zero.

Note: In the expressions above, the correlation coefficients among the multipath components and the correlation coefficients among the shadowing components are not explicitly related to the multipath fading and shadowing parameters, respectively. This assumption is valid for shadowing correlations where the correlation coefficient is dependent on the distance between the receiving antennas and possibly on the angle of arrival [8] and not related to the standard deviation ( $m_{s}$ parameter) [18]. For multipath fading, the multipath components, for geographically distributed antenna ports, are typically uncorrelated.

\section{Approximating the PDF of the Sum of IDENTICALLY DISTRIBUTED AND POSITIVELY CORRELATED GENERALIZED- $K$ RVS}

The derivation of the PDF of $\xi_{1}$ is an analytically difficult problem. As an alternative, we may consider approximating the PDF of $\xi_{1}$ by an appropriate distribution using the moment matching method. So, in this section, the approximation of the PDF of the sum of identically distributed $N$ generalized- $K$ RVs whose shadowing components are equally correlated by the 
familiar Gamma PDF is proposed. The choose of the Gamma distribution is motivated by the following (i) The Gamma PDF is a candidate for approximating the PDF of the sum of positive RVs whenever the central limit theorem is not applicable which includes the sum of correlated positive RVs, (ii) the generalized- $K$ distribution approaches a Gamma distribution for large $m_{m}$ and/or $m_{s}$ [3] and the sum of correlated Gamma RVs has been approximated by a single Gamma RV using the moment matching method [19, 20]; and (iii) the Gamma distribution is a tractable model that allows the derivation of closed-form expressions for the different performance metrics [21]. The other candidate approximating distributions such as the Gumbel and generalized Weibull distributions [22] are not as beneficial as the Gamma distribution.

Proposition: The PDF of the sum of $N$ identically distributed generalized- $K$ RVs whose shadowing components are equally correlated can be approximated, for a small number of summands, by the PDF of a Gamma RV, $\xi$ [23],

$$
p_{\xi}(x)=\frac{\theta^{-k}}{\Gamma(k)} x^{k-1} \exp \left(-\frac{x}{\theta}\right),
$$

whose shape and scale parameters can be expressed as

$$
\begin{gathered}
k=\frac{1}{\frac{1}{N m_{m}}+\frac{1-\rho_{s}}{N m_{s}}+\frac{1}{N m_{m} m_{s}}+\frac{\rho_{s}}{m_{s}}-\epsilon}, \\
\theta=\frac{N \Omega_{0}}{k} .
\end{gathered}
$$

Proof: The proof is based on obtaining a Gamma PDF whose $A F$ equals $A F_{\xi_{1}}$. So, equating the $A F$ of the approximating Gamma distribution to $\mathrm{AF}_{\xi_{1}}$ as

$$
\mathrm{AF}_{\xi}=1 / k=\frac{1}{N m_{m}}+\frac{1-\rho_{s}}{N m_{s}}+\frac{1}{N m_{m} m_{s}}+\frac{\rho_{s}}{m_{s}}
$$

results in the expression in (20-a). The expression in (20b) is simply obtained by matching the first moment. The appropriate value of the adjustment parameter $\epsilon$ can be determined numerically by minimizing the absolute value difference between the approximating and approximated PDFs and can be tabulated ${ }^{2}$.

\section{ON THE AF FOR THE SUM OF NON-IDENTICALly DisTRIBUTED CORRELATED GENERALIZED- $K$ RVS}

A general expression of the correlation coefficient for nonidentically distributed (n.i.d.) correlated generalized- $K$ RVs with arbitrarily different values of $m_{m}, m_{s}$ and $\Omega_{0}$ can be obtained by substituting (9) in (15). However, assuming that $\Omega_{0,1}=\Omega_{0,2}=\ldots .=\Omega_{0, N}=\Omega_{0}$, the AF becomes

$$
\begin{aligned}
& \mathrm{AF}_{\xi_{1}}=\frac{\sum_{i=1}^{N} \frac{\left(m_{m, i}+m_{s, i}+1\right)}{m_{m, i} m_{s, i}}}{N^{2}} \\
& +\frac{\sum_{i=1}^{N} \sum_{j=1, i \neq j}^{N}\left[\frac{\rho_{w_{i}, w_{j}}}{\sqrt{m_{s, i} m_{s, j}}}+\frac{\rho_{z_{i}, z_{j}}}{\sqrt{m_{m, i} m_{m, j}}}+\frac{\rho_{w_{i}, w_{j}} \rho_{z_{i}, z_{j}}}{\sqrt{m_{m, i} m_{m, j}} \sqrt{m_{s, i} m_{s, j}}}\right]}{N^{2}} .
\end{aligned}
$$

\footnotetext{
${ }^{2} \mathrm{~A}$ similar approach was used in [24] to approximate, region-wise, the generalized- $K$ PDF by a Gamma PDF. More details are given in [25].
}

Furthermore, when shadowing correlations only are present, $\rho_{i, j}$ reduces to

$$
\rho_{i, j}=\frac{\rho_{w_{i}, w_{j}} \sqrt{m_{m, i} m_{m, j}}}{\sqrt{\left(m_{m, i}+m_{s, i}+1\right)} \sqrt{\left(m_{m, j}+m_{s, j}+1\right)}},
$$

which results in

$$
\mathrm{AF}_{\xi_{1}}=\frac{\sum_{i=1}^{N} \frac{\left(m_{m, i}+m_{s, i}+1\right)}{m_{m, i} m_{s, i}}+\sum_{i=1}^{N} \sum_{j=1, i \neq j}^{N} \frac{\rho_{w_{i}, w_{j}}}{\sqrt{m_{s, i} m_{s, j}}}}{N^{2}} .
$$

\section{RESUlts AND Discussions}

In this section, results for the relevant physical scenario where the multipath components are uncorrelated and the shadowing are correlated with $\rho_{s} \approx 0.5$ are presented. The plot of the correlation coefficient, $\rho_{i, j}$, as given in (13) for $\rho_{m}=0, \rho_{s}=0.5$, and different values of $m_{m, i}=m_{s, i}=m_{1}$ and $m_{m, j}=m_{s, j}=m_{2}$ is shown in Fig. 1. The main observation is that the magnitude of $\rho$ is upper bounded by $\rho_{s} / 2$ and decreases as $m_{m}$ and/or $m_{s}$ decreases. In fact, for the identically distributed case, $\left(m_{1}=m_{2}=m\right)$, we may write $\rho_{i, j}=\frac{\rho_{s} m}{2 m+1}$ which clearly approaches $\rho_{s} / 2$ as $m$ gets large.

The CDF plots for the sum distribution and the approximating Gamma distribution are shown in Fig 2 for $\rho_{m}=0, \rho_{s}=$ 0.5 and $m=m_{m}=m_{s}=2$. Now, since the diversity gain, $d_{g}$ for the Gamma model can be well-approximated for moderate and large signal-noise ratio (SNR) by the AF [11], we may express the approximate diversity gain as $d_{g}=\frac{1}{\mathrm{AF}_{\xi_{1}}-\epsilon}$ for $N=2$, and 4 , and $\rho_{s}=0.5$. The corresponding values of the adjustment parameter for $m=2$, in Fig. 2, are $\epsilon=0.22$ and $\epsilon=0.04$ for $N=2$ and $N=4$, respectively.

Note: As the $m_{s}$ parameter increases, the correlation coefficient between the two generalized- $K \mathrm{RV}$ s decreases; as a consequence, the PDF of sum distribution is better approximated by the PDF of another generalized- $K \mathrm{RV}$ whose parameters are obtained by the moment matching method as shown in Fig. 3.

\section{CONCLUSION}

The generalized- $K$ model has been recently proposed to model composite fading in wireless channels. Hence the knowledge the distribution of the sum of generalized- $K \mathrm{RVs}$ is needed for performance analysis over such channels especially for the correlated case since correlations among shadowing components occur in realistic propagation scenarios. In this paper, the expression of the amount of fading for correlated generalized- $K$ composite fading channels is developed and the PDF of the sum of identically distributed generalized- $K$ RVs with equally correlated shadowing components is approximated by the tractable Gamma distribution. The obtained results have shown that the PDF of the sum can be closely approximated by the PDF of a Gamma RV which highlights new insights into role of shadowing correlations in the gain of MRC in composite fading channels. 


\section{REFERENCES}

[1] D. J. Lewinsky, "Nonstationary probabilistic target and clutter scattering models," IEEE Trans. Antenna and Propag., vol. AP-31, no. 3, pp. 490498, May 1983.

[2] P. M. Shankar, "Error rates in generalized shadowed fading channels," Wireless Personal Commun., vol. 28, no. 4, pp. 233-238, Feb. 2004

[3] P. M. Kostic, "Analytical approach to performance analysis for channel subject to shadowing and fading," IEE Proc. Commun., vol. 152, no. 6, pp. 821-827, Dec. 2005

[4] P. S. Bithas, N. C. Sagias, P. T. Mathiopoulos, G. K. Karagiannidis and A. A. Rontogiannis, "On the performance analysis of digital communications over generalized-K fading channels," IEEE Commun. Lett., vol. 5, no. 10, pp. 353-355, May 2006.

[5] P. S. Bithas, P. T. Mathiopoulos, and S. A. Kotsopoulos, "Diversity reception over generalized- $K\left(K_{G}\right)$ fading channels," IEEE Trans. Wirel. Commun., vol. 6, no. 12, pp. 4238-4243, Dec. 2007.

[6] P. M. Shankar, "Outage probabilities of a MIMO scheme in shadowed fading channels with micro- and macrodiversity reception," IEEE Trans. Wireless Commun., vol. 7, no. 6, pp. 2015-2019, June 2008.

[7] A. Conti, M. Z. Win, and M. Chiani, "Slow adaptive $M$-QAM with diversity in fast fading and shadowing," IEEE Trans. Commun., vol. 55, no. 5, pp. 895-905, May 2007.

[8] F. Graziosi and F. Santucci, "A general correlation model for shadow fading in mobile radio systems", IEEE Commun. Lett., vol. 6, no. 3, pp. 102-104, Mar. 2002.

[9] B. Holter and G. E. Øien, "On the amount of fading in MIMO diversity systems," IEEE Trans. Wirel. Commun., vol. 4, no. 5, pp. 2498-2507, Sep. 2005.

[10] Y. Li and S. Kishore, "Diversity factor-based capacity asymptotic approximations of MRC reception in Rayleigh fading channels,"IEEE Trans. Commun., vol. 56, no. 6, pp. 858-861, June 2008.

[11] Z. Wang and G. B. Giannakis, "A simple and general parameterization quantifying performance in fading channels," IEEE Trans. Commun., vol. 51, no. 8, pp. 13891398, Aug. 2003.

[12] A. Abdi and M. Kaveh, "On the utility of Gamma PDF in modeling shadow fading (slow fading)," in Proc. IEEE Veh. Technol. Conf., vol. 3, pp. 2308-2312, May 1999.

[13] M. D. Springer and W. E. Thompson, "The distribution of products of Beta, Gamma and Gaussian random variables," SIAM Journal of Applied Maths., vol. 18, pp. 721-737, 1970.

[14] A. Charash, "A study of multipath reception with unknown delays," PhD dissertation, University of California, Berkeley, CA, Jan. 1974.

[15] E. Perahia, D. C. Cox, and S. Ho, "Shadow fading cross correlation between basestations,", in Proc. IEEE Veh. Technol. Conf., pp. 2308-2312, May 2001

[16] J. E. Prussing, "The principal minor test for semidefinite matrices," $J$. Guidance., vol. 9. no. 1, pp. 121-122, Jan.-Feb. 1986

[17] C. D. Meyer, Matrix Analysis and Applied Linear Algebra, 2nd ed Philadelphia, PA: SIAM, 2000

[18] Z. Wang, E. K. Tameh, and A. R. Nix, "Joint shadowing process in urban peer-to-peer radio channels," IEEE Trans. Veh. Technol., vol. 57, no. 1, pp. 52-64, Jan. 2008.

[19] S. Kotz and J. Adam, "Distribution of sum of identically distributed exponentially correlated gamma variables,", Annals of Math Statistics, vol. 35. pp. 227-283. 1964.

[20] V. A. Aalo, "Performance of maximal-ratio diversity systems in a correlated Nakagami-fading environment", IEEE Trans. Commun., vol. 43, no. 8, pp. 2360-2369, Aug. 1995.

[21] M. K. Simon and M.-S. Alouni, Digital Communication over Fading Channels. 2nd Ed. New York: Wiley, 2005.

[22] E. Bertain and M. Clusel, "Generalized extreme value statistics and sum of correlated variables," J. Phys. A:Math. Gen., vol. 39, pp. 7607-7619, 2006.

[23] A. Papoulis, Probability, Random Variables, and Stochastic Processes. 2nd Ed. New York: McGraw-Hill, 1984.

[24] S. Al-Ahmadi and H. Yanikomeroglu,"On the approximation of the generalized-K PDF by a Gamma PDF using the moment matching method," in Proc. IEEE Wireless Commun. Networking Conf. (WCNC 2009), pp. 1-6, April 2009.

[25] S. Al-Ahmadi and H. Yanikomeroglu, "On the approximation of the generalized- $K$ distribution by a Gamma distribution for modeling composite fading channels," IEEE Trans. Wirel. Commun., vol. 9, no. 2, pp. 706-713, Feb. 2010.

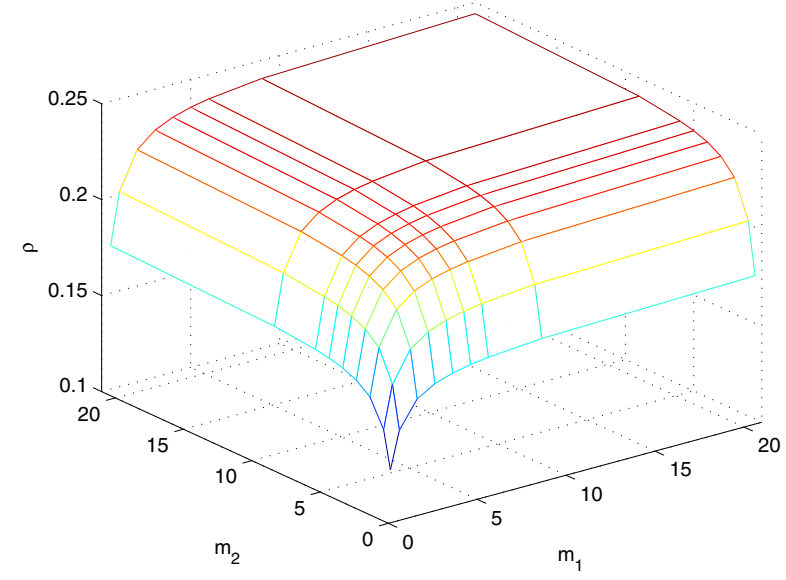

Fig. 1. The plot for the correlation coefficient between two generalized- $K$ $\mathrm{RV}$ s as a function of their multipath and shadowing parameters.

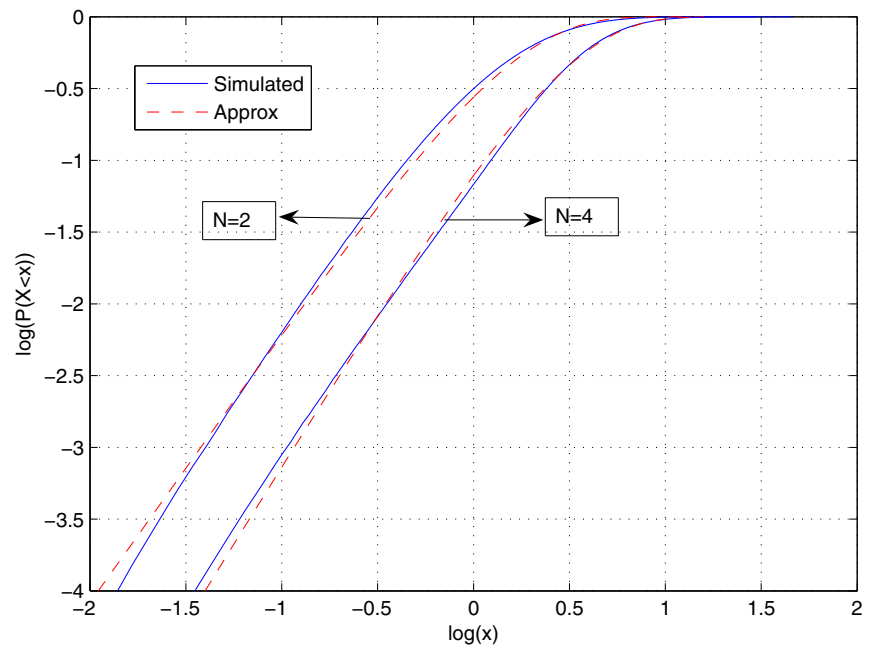

Fig. 2. The CDF plots for the sum of correlated generalized- $K$ RVs and the approximating Gamma RV for different values of $N$ with $m_{m}=m_{s}=2$.

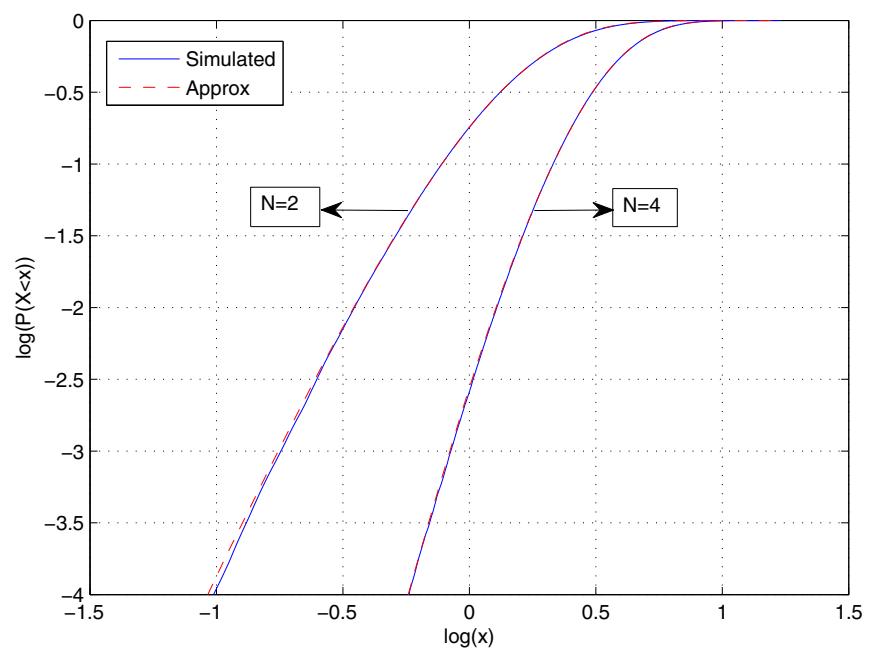

Fig. 3. The CDF plots for the sum of correlated generalized- $K$ RVs and the approximating generalized- $K \mathrm{RV}$ for different values of $N$ with $m_{m}=2$ and $m_{s}=9$. 